\title{
A device for automated hydrodynamic shearing of genomic DNA
}

\author{
Aric Joneja and Xiaohua Huang \\ Department of Bioengineering, University of California, San Diego, \\ La Jolla, CA, USA
}

BioTechniques 46:553-556 (June 2009) doi 10.2144/000113123

Keywords: hydrodynamic shear stress; DNA shearing; genomic DNA fragmentation; genomic DNA libraries; genome sequencing; filter screen

Supplementary material for this article is available at www.BioTechniques.com/article/113123.

We describe a device for automated fragmentation of genomic DNA by hydrodynamic shearing using a filter screen with uniform pores. Human genomic DNA can be fragmented reproducibly to a range of 2000-12,000 bp by using various fluid flow rates and screens with $0.5-10-\mu \mathrm{m}$ pores. The utilization of disposable screens eliminates sample cross-contamination and the tendency of device clogging, commonly encountered in singleorifice shearing devices.

The fragmentation of chromosomal DNA and production of unbiased genomic DNA libraries is a critical and sometimes rate-limiting step in the DNA sequencing pipeline, especially for many next-generation sequencing platforms. Currently, four methods are commonly used for DNA fragmentation: enzymatic digestion (1-7), sonication (8), nebulization (9-11) and hydrodynamic shearing $(12,13)$. Even though all have been used in library construction, these methods have limitations. Examples include the biases produced by endonuclease digestion, low cloning efficiency due to DNA damage by sonication $(12,14,15)$, large size distribution and difficulty in automation by nebulization, and the high cost and clogging issues by hydrodynamic shearing using instruments with single-orifice devices.

The hydrodynamic shearing method is widely used for shearing genomic DNA $(12,13)$. The DNA molecules are fragmented by hydrodynamic shear stress when they are forced through a point-sink such as a small orifice or the bore of a smalldiameter tube at high velocity. Genomic DNA can be sheared randomly with a tight 2-3-fold size distribution, and the cloning efficiency of the sheared DNA fragments is very high $(12,13)$. The length of the fragments is determined by the flow velocity of the solution and the diameter of the orifice, independent of the initial DNA size/concentration and salt concentration
(13). A commercial instrument and various shearing assemblies containing a single laserdrilled orifice on a ruby jewel (Hydroshear; Genomic Solutions Inc., Ann Arbor, MI, USA) are available for performing the process semi-automatically. However, the instrument is expensive for many investigators. The shearing assemblies also tend to get clogged and are too expensive to be disposed of after every use.

We describe an inexpensive device for automated fragmentation of genomic DNA using hydrodynamic shearing. A schematic of the device is illustrated in Figure 1. The system consists of a computer-controlled syringe pump with a built-in 9-port valve and a filter screen housed in an adaptor connected to the valve via rigid tubing. The key component of the device is a disposable stainless steel filter screen (VICI-Valco Instruments Co. Inc., Houston, TX, USA), which contains thousands of uniform pores with micron or sub-micron dimensions, in contrast to the single-orifice devices used in the shearing assemblies of the Hydroshear instrument. Similar to the Hydroshear instrument, hydrodynamic shear stress is utilized to break long DNA molecules into smaller fragments.

Shearing genomic DNA with our device entails a washing step and a shearing step that can be carried out automatically in $\sim 20$ $\mathrm{min}$. First, the desired screen is inserted into the adaptor and the fluidic system is washed extensively with $\mathrm{HCl}, \mathrm{NaOH}$, water, and a buffer. Second, the DNA is loaded and sheared by running the solution through the screen 20 times. A computer-controlled syringe pump with a 9-port valve is used to fully automate the entire shearing process. We have shown that human genomic DNA can be sheared using screens with pore sizes of $0.5 \mu \mathrm{m}, 1 \mu \mathrm{m}, 2 \mu \mathrm{m}$, and $10 \mu \mathrm{m}$ at fluid flow rates of $15-125 \mathrm{~mL} / \mathrm{min}$. Table 1 lists the average lengths and distributions of the fragments and the coefficients of variation $(\mathrm{CVs})$ from three independent runs using 3 different $0.5-\mu \mathrm{m}$ and $10-\mu \mathrm{m}$ screens at 6 different flow rates. Typical gel images of the sheared products are shown in Supplementary Figure 1, A and B. Shearing with screens with $1-\mu \mathrm{m}$ and $2-\mu \mathrm{m}$ pore sizes gave results similar to those obtained with the $0.5-\mu \mathrm{m}$ screen. Detailed information on the materials, methods, and analysis is provided in the Supplementary Materials.

The average length of the fragments is controlled primarily by the fluid flow rate, the size of the pores in the screen, and the iterations of sample passing through the screen. As shown in Supplementary Figure 1C, the majority of DNA molecules were sheared to the target size in just one iteration. Almost all DNA fragments approached the minimal length after 5-10 iterations. No further change in length and distribution was observed after 20 iterations. The more important parameters are the flow rate and screen pore size. The fluid flow rates given in the text and the figures are the speeds at which the syringe pump delivers the fluid, not the actual flow rates of the DNA solution through the pores. The screen is constructed of woven stainless steel fibers, and the pores are of irregular geometry but are very uniform in shape and size across the entire screen (Supplementary Figure 2). Due to the complex geometry of the pores, the flow rate of the fluid through each pore increases as the fluid enters, reaches the maximum as the fluid reaches the smallest cross-section, and then decreases as the fluid leaves the pore. We have estimated that the pores account for $\sim 2 \%$ and $\sim 10 \%$ of the crosssection of the $0.5-\mu \mathrm{m}$ and $10-\mu \mathrm{m}$ screens, respectively, so the actual flow rate is respectively $50 \times$ and $10 \times$ that delivered by the syringe pump. The size of the pores is vast $(\geq 500 \mathrm{~nm})$ compared with the diameter of the DNA molecule $(2 \mathrm{~nm})$, so the effect of pore geometry on the shear stresses on the DNA molecules is expected to be minimal. With the exceptional uniformity in size, geometry, and distribution across each screen and between different batches of screens, a narrow range of fragment sizes with $>80 \%$ of fragments within a 2 - to 3 -fold range can be achieved reproducibly. 


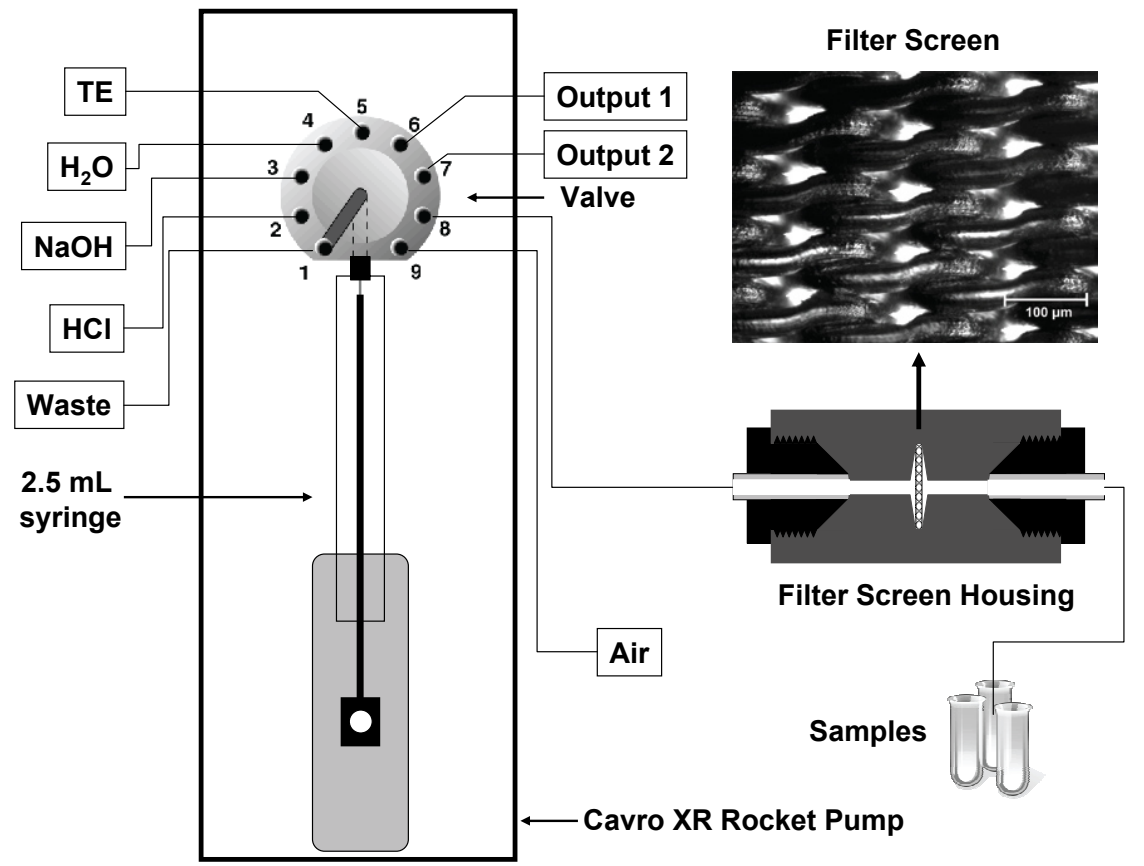

Figure 1. Schematic of a device for hydrodynamic shearing of DNA. A Cavro XR pump with a built-in 9-port valve is used. A cross-section of the filter screen housing is also illustrated. Shown at the upper right corner is a transmitted-light micrograph of a portion of a $10-\mu \mathrm{m}$ screen. The computer used to control the instrument is not shown.

A

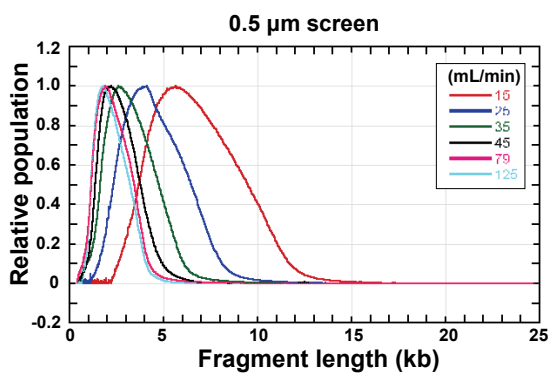

B

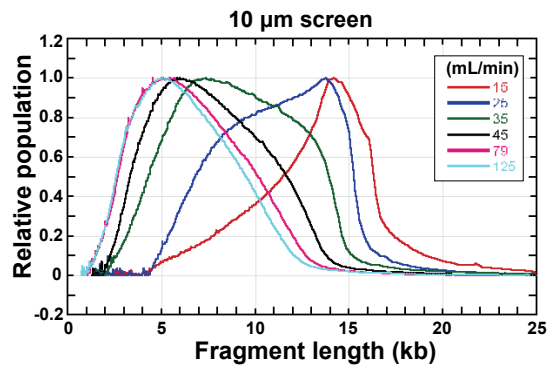

C

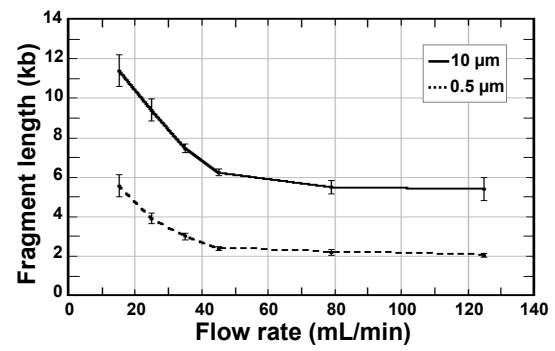

Figure 2. Lengths and size distributions of the DNA fragments as a function of screen pore size and flow rate. Distribution of DNA fragments produced by shearing with screens of (A) $0.5-\mu \mathrm{m}$ and (B) $10-\mu \mathrm{m}$ pore sizes at various flow rates, plotted as relative population of the DNA fragments as a function of fragment size. The slight distortion of the curves at larger fragment sizes seen in (B) could be due to artifacts in the gel analysis of large DNA fragments. (C) Fragment lengths versus fluid flow rates. Human genomic DNA was sheared at six different speeds using screens with pore sizes of $0.5 \mu \mathrm{m}$ and $10 \mu \mathrm{m}$. The products were separated by electrophoresis with $0.8 \%$ agarose gel and the average fragment size was computed as described in the Supplementary Materials. Data from three shearing runs with the same screen were averaged to obtain the data. Each error bar is one standard deviation from the mean and is intended to demonstrate reproducibility across 3 shearing runs.

Table 1. Flow Rates and Fragment Lengths: Reproducibility between Different Batches of Screens

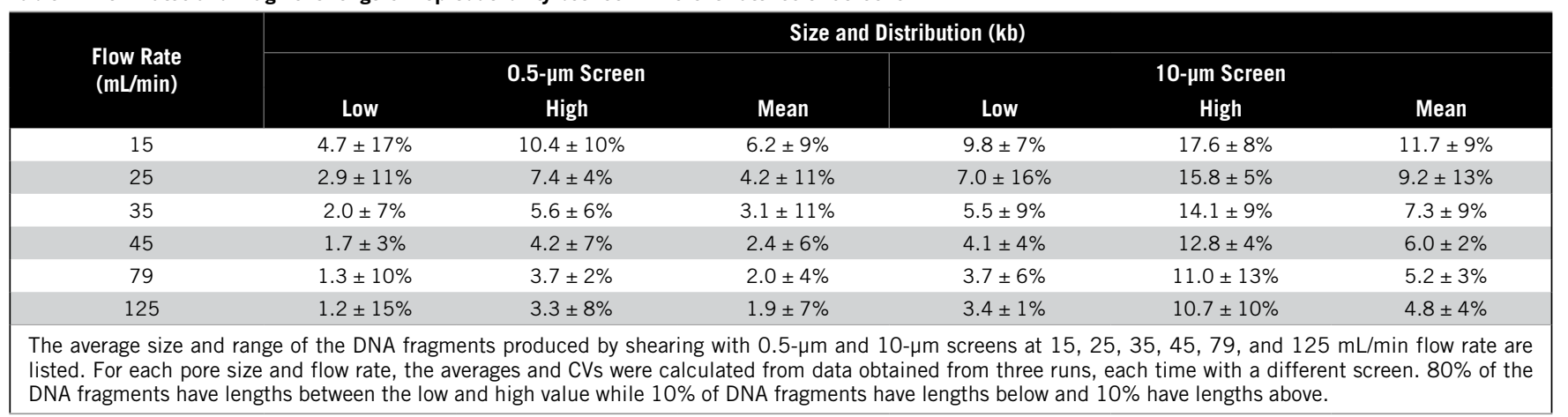


In all cases, a higher flow rate resulted in smaller DNA fragments, and smaller pore size produced shorter fragments at all fluid flow velocities. The CV was usually small $(<10 \%$ between three runs with the same screen or three different batches of screens).

Figure 2, $A$ and $B$ show the relationships between the flow rates and lengths of the DNA fragments produced by shearing with $0.5-\mu \mathrm{m}$ and $10-\mu \mathrm{m}$ screens. Fragment sizes of $2-12 \mathrm{~kb}$ can be achieved using screens of these two pore sizes by varying the flow rates. The minimum average size that can be produced with our current device is $\sim 2000$ bp using a $0.5-\mu \mathrm{m}$ screen (the smallest pore size currently available from VICIValco) with a flow rate of $125 \mathrm{~mL} / \mathrm{min}$ (the maximum flow rate attainable with our current system). As shown in Figure 2C, the nonlinear relationship between flow rate and fragment size follows a power law. The increase in flow rate from $40 \mathrm{~mL} / \mathrm{min}$ to $125 \mathrm{~mL} / \mathrm{min}$ resulted in only a small decrease in fragment size. Shearing DNA to fragments $<2000 \mathrm{bp}$ will require screens of smaller pore sizes and a syringe system that can deliver higher flow rates. Very little heat is generated in our shearing process, but, as a precaution against any potential GC bias (16), the shearing assembly can be cooled on ice as usually practiced in the DNA shearing process.

A major concern for hydrodynamic shearing instruments that employ singleorifice devices is the frequent clogging of the small orifice. Our attempts to design a custom shearing instrument were prompted by multiple failed attempts to shear DNA with the small HydroShear-Custom Shearing Assembly (650-5000 bp). Despite thoroughly filtering all reagents, clogging of the orifice frequently terminated our experiments prematurely. With the use of a screen, however, we never experienced any clogging. The screens can be used repeatedly without any change in performance,which makes our system more robust and userfriendly. No noticeable evidence of rust was observed on the stainless steel screens even after several weeks of use, but if rust is a concern, filters made of PEEK-encapsulated titanium (available from VICI-Valco) can be used. Another concern is potential sample carryover contamination. The shearing assemblies used in the Hydroshear instrument are too expensive to be disposed of after every use. We found that with our standard wash procedure, reusing the screens increases the potential of sample carryover, which can be detected by PCR amplification (Supplementary Figure 3). Fortunately, the screens used in our device are very inexpensive (\$2 USD) and can be replaced easily. Cross-contamination can be eliminated entirely by replacing the screens between samples.

It has been shown that DNA fragments obtained by hydrodynamic shearing are random and have high cloning efficiency; thus, they are suitable for genomic library construction $(12,13)$. Since our device utilizes the same hydrodynamic shearing mechanism, it is reasonable to expect that the fragments produced using our device are random and have similar high cloning efficiency.

In summary, we have discovered that stainless steel filter screens with very uniform pores can be used in place of singleorifice devices to shear genomic DNA. We have developed an inexpensive device and the procedure for fully automated hydrodynamic shearing of genomic DNA with comparable performance to that of the Hydroshear instrument $(12,13)$. The utilization of the inexpensive disposable screens eliminates potential sample crosscontamination and device clogging. Our device can be assembled easily from very inexpensive and commercially available parts. We believe our significantly improved device and method for hydrodynamic shearing of DNA will be of great utility in producing genomic DNA libraries for genome sequencing using nextgeneration sequencing platforms.

\section{Acknowledgments}

We thank Kristopher D. Barbee for taking the SEM images of the stainless steel filter screens. This work was supported in part by the National Institutes of Health (NIH; grant nos. HG003587 and HG004130) and the National Science Foundation (grant no. BES-0547193, a CAREER Award to X.H). This paper is subject to the NIH Public Access Policy.

The authors declare no competing interests.

\section{References}

1. Anderson, S. 1981. Shotgun DNA sequencing using cloned DNase I-generated fragments. Nucleic Acids Res. 9:3015-3027.

2. Roe, B.A. 2004. Shotgun library construction for DNA sequencing. Methods Mol. Biol. 255:171-187.

3. Seed, B., R.C. Parker, and N. Davidson. 1982. Representation of DNA sequences in recombinant DNA libraries prepared by restriction enzyme partial digestion. Gene 19:201-209.

4.Gingrich, J.C., D.M. Boehrer, and S.B. Basu. 1996. Partial CviJI digestion as an alternative approach to generate cosmid sublibraries for large-scale sequencing projects. BioTechniques 21:99-104.

5. Hoheisel, J.D., D. Nizetic, and H. Lehrach. 1989. Control of partial digestion combining the enzymes dam methylase and Mbol. Nucleic Acids Res. 17:9571-9582.

6. Wong, K.K., L.M. Markillie, and J.D. Saffer. 1997. A novel method for producing partial restriction digestion of DNA fragments by PCR with 5-methyl-CTP. Nucleic Acids Res. 25:4169-4171.

7. Osoegawa, K., A.G. Mammoser, C. Wu, E. Frengen, C. Zeng, J.J. Catanese, and P.J. de Jong. 2001. A bacterial artificial chromosome library for sequencing the complete human genome. Genome Res. 11:483-496.

8. Deininger, P.L. 1983. Approaches to rapid DNA sequence analysis. Anal. Biochem. 135:247-263.

9. Okpodu, C.M., D. Robertson, W.F. Boss, R.K. Togasaki, and S.J. Surzycki. 1994. Rapid isolation of nuclei from carrot suspension culture cells using a BioNebulizer. BioTechniques 16:154-159.

10. Hengen, P.N. 1997. Shearing DNA for genomic library construction. Trends Biochem. Sci. 22:273-274.

11. Burger, G., D.V. Lavrov, L. Forget, and B.F. Lang. 2007. Sequencing complete mitochondrial and plastid genomes. Nat. Protocols 2:603-614.

12.Oefner, P.J., S.P. Hunicke-Smith, L. Chiang, F. Dietrich, J. Mulligan, and R.W. Davis. 1996. Efficient random subcloning of DNA sheared in a recirculating point-sink flow system. Nucleic Acids Res. 24:3879-3886.

13. Thorstenson, Y.R., S.P. HunickeSmith, P.J. Oefner, and R.W. Davis. 1998. An automated hydrodynamic process for controlled, unbiased DNA shearing. Genome Res. 8:848-855.

14. Bertazzoni, U. 1975. Analysis of the breaking sites in the physical degradation of DNA. Biochim. Biophys. Acta 395:239-245.

15. McKee, J.R., C.L. Christman, W.D. O'Brien, Jr., and S.Y. Wang. 1977. Effects of ultrasound on nucleic acid bases. Biochemistry 16:4651-4654.

16. Roe, B.A., J.S. Crabtree, and A.S. Khan. 1996. DNA Isolation and Sequencing. John Wiley \& Sons, New York.

Received 12 November 2008; accepted 2 February 2009.

Address correspondence to Xiaohua Huang, Department of Bioengineering University of California, San Diego, 9500 Gilman Drive, La Jolla, CA, 92093, USA. e-mail: x2huang@ucsd.edu 